\title{
INDICADORES DAS CONDIÇÕES NUTRICIONAIS NA REGIÃO DO POLONOROESTE. I. ASPECTOS METODOLÓGICOS E CARACTERÍSTICAS SÓCIO-ECONÔMICAS (1)
}

\author{
M.A. dos SANTOS SPINELII (2), R.R. da SII.VA (2), M.C.W. ALBUQUERQUE (3), \\ E.M. YOKOO (3) \& I.V. GUIMARÃES (4)
}

\begin{abstract}
RESUMO
Otrabalho apresenta os principios metodológicos que nortearam a organização da Pesquiva Inquéto Nuricional no Polonoroeste. Foram incluídas, também as informacoes sobre as caractésticas sócio-economicas e sanitálas das famílias estudadas em 1985 na yona urbana de Cáceres $\mathrm{MT}$. Destas $74 \%$ eram radicadas em Cáceres $13,5 \%$ nrentiam de outros esados ou de outros paises, sendo que $76,2 \%$ residam on zona urtara. Cuanto a categoria social e renda $52 \%$ eram urbanos e $51,0 \%$ recbla nenos ta 2 starios minmos. O inquerto nào aingia os 2 setores mais pobies e populoson da cidade onde as condiçôes sāo piores e onde vive a maioria da populaçào que migrou mais recontemente para Caceres.
\end{abstract}

MTERMOS: Inquerio Nubchonat-Metodologia; Caracteristicas sócio-ecomonicat.

\section{INTRODUÇÃO}

A pesquisa Diagnóstico em Saúde na regiāo do Polonoroeste desenvolvida por um grupo de pesquisadores do Centro de Ciencias Biológicas e da Saúde - Universidade Federal de Mato Grosso tem como objetivo acompanhar a evolução das condições de saúde na área de influência da Rodovia Cuiabá-MT - Porto Vethe, RO, situada no Sudoeste do Estado de Mato Grosso, antes, durante e depois da implantação do Projeto Polonoroeste que previa assentamentos agricolas, asfaltamento de estradas vicinais e projetos na área de Educação, Saúde e Agricultura, através do Programa de Desenvolvimento Rural Integrado (PDRI).

A região em estudo é constituída por 3 ecossistemas distintos: cerrado, pantanal e mata galeria (redução gradativa da floresta amazônica). Além das cidades nascidas no processo histórico de ocupação do Estado de Mato Grosso no período colonial, a maior parte da região passou a ser ocupada a partir da década de 50 quando se iniciou a expansão da fronteira agrícola para a região Centro-Oeste. Esta expansão resultou na criação de núcleos de colonização

(1) Pesquisa realizada com recussos do Convênio n: 700. 1020.0/83 - CNPq-UFMT-SES e 700.405/85 CNPq-UFMT-SES.

(2) Professor do Depantamento de Nutriçäo-UPMT e Pesquisador do Grupo Inquérito Nutricional no Polonoroeste - MT.

(3) Professor-Auxiliar do Departamento de Nutricăo-UnMT e Pesquisador do Grupo Inquérito Nutricional no Polonoroeste-NT.

(4) Bolsista-Supervisora do Cinupo Inquérito Nuricional no Polonoroeste-MT.

Enderefo para correspondència: Universidade Federal de Malo Grosso - Av. Fernando Corrêa, $5 / n$ ? - 78100, Cuiabá, Mato Grosso, Brasil. 
SANTOS SPINELLI, M.A. dos; SILVA, R.R. da; ALBUQUERQUE, M.C.W.; YOKOO, E.M. \& GUIMARÃES, L.V. - Indicadores das condiçós nutricionais na Regiāo do Polonoroeste. I. Aspectos metodologicos e característicás sócio-econômicas. Rev. Inst. Med. trop. São Paulo, 30(3): 169-173, 1988 .

tanto da iniciativa privada quanto pública que deram origem na década de 70 , a diversos dos municipios da regiào.?

Os dados censitários de 1980 revelam que $34,6 \%$ da população desta região são oriundos de outros Estados do Brasil e, segundo análises da Fundação de Pesquisa Cândido Rondon (MT) muitos dos migrantes, não alcançando equilibrio econômico no meio rural, estabeleceram-se na periferia dos núcleos urbanos da região o que tem gerado um crescimento desordenado das cidades que não contam com infra-estrutura de serviços adequada. Verificase que $68,8 \%$ da população total na área vive em zona urbana. 5

Sabendo-se que as condições de Saúde Doença são resultantes destes processos gerais a nivel de estrutura econômica e social e, que o perfil epidemiológico da região é pouco conhecido, propôs-se, para o alcance dos objetivos da Pesquisa, uma investigação abrangendo um amplo espectro de informações que podem ser assim categorizadas:

a) inquérito epidemiológico das doenças mais prevalentes na regiâo, do estado nutricional e das condições sanitárias do domicílio e peri-domiciliares.

b) estudo da estrutura dos Serviços de Saúde.

c) estudo das condições sócio-econômicas. ${ }^{3}$

Para o encaminhamento da Pesquisa, financiada pelo CNPq (Conselho Nacional de Pesquisa) em convênio com a Universidade Federal de Mato Grosso e a Secretaria Estadual de Saúde - MT, foram elaborados os seguintes projetos:?

1) Levantamento Epidemiológico por Entrevistas Domiciliares.

2) Inquérito Nutricional.

3) Investigação Epidemiológica por exames clínicos.

4) Investigação Epidemiológica por exames laboratoriais.

5) Estudo de Hipertensão Arterial.

6) Estudo dos Serviços de Saúde.

Foram realizados 3 levantamentos de campo: em 1983, 1985 e 1986; o de 1983 investigou as áreas urbanas dos municípios de Jauru, Ara- putanga, Mirassol D'Oeste, Cáceres, Tangará da Serra e do Distrito de Nova Olímpia; os de 1985 e 1986 foram realizados na sede do municipio de Cáceres.

Para a caracterização das Condições de Nutrição e Alimentação o grupo do Inquérito Nutricional partiu da premissa que a desnutrição, expressão biológica da fome, fenômeno social, é mais freqüentemente evidenciada em crianças de até 5 anos de idade, e é resultado direto da oferta de alimentos no domicílio, que, por sua vez, "depende fundamentalmente da capacidade que a familia tem de obter quantidades adequadas de alimentos" 4 , e é a inserção do individuo no processo produtivo que determinará esta capacidade. +

Deste modo o estudo Inquérito Nutricional abrangerá 3 partes:

a) As condições nutricionais podem ser facilmente avaliadas através das medidas do corpo, mesmo nos estágios mais precoces das deficiências alimentares, indicou-se, assim a necessidade de se reconhecer o estado nutricional das crianças através de um estudo antropométrico privilegiando as crianças de 3 a 72 meses de idade. No estudo de 1983 foi também realizada uma investigação clíniconutricional a fim de caracterizar as manifestaçôes clínicas da desnutrição.

b) A investigação sobre o consumo de alimentos seria fonte de referência para identificar as condições de alimentação. No primeiro estudo, em 1983, esta investigaçâo foi realizada no âmbito das famílias às quais pertenciam as crianças avaliadas pelo estudo antropométrico. Nos levantamentos de $1985 \mathrm{e}$ 1986, procurou-se caracterizar processo de amamentação e desmame das crianças de 6 a 24 meses de idade, incluídas na amostra, além da sua alimentação no momento da pesquisa.

c) As investigações de 1985 e 1986 buscaram identificar algumas variáveis que permitissem caracterizar as famílias da amostra quanto às condições da habitação e quanto à ocupação, posição na ocupação e renda do chefe da familia.

Os dados dos estudos clínicos, antropométricos e de consumo alimentar são apresentados em olitros artigos desta mesma publicação. 
SANTOS SPINELll, M.A. dos; SILVA, R.R. da; ALBUQUERQUE, M.C.W.: YOKOO, E.M. \& GUIMARÄES. L.V. - Indicadores das condiçỏes nutricionais na Região do Polonoroestc. I. Aspectos meiodológicos e características sócio-econômicas. Rev. Inst. Med. trop. São Paulo, 30(3): 169-173, 1988

\section{MATERIAL E METODO}

\section{AMOSTRAS}

O sistema de amostragem elaborado para o Projeto "Pesquisa em Saúde Pública, no Polonoroeste" em Mato Grosso, seguiu o esquema clássico de "Amostragem por Área", estabelecido na Pesquisa Nacional por Amostra de Domicilio (PNAD), modificado por CARVAL.HEIRO \& SANCHES". O modelo amostral adotado foi estratificado em múltiplos estágios, buscando representar os sistemas ecológicos e as diversidades econômicas e sociais que caracterizam esta área $6.200 \mathrm{~km}$ ?

Os estudos dietéticos e antropométricos utilizaram nos 3 levantamentos o modelo amostral do "Levantamento Epidemiológico por Entrevistas Domiciliares", trabalhando em uma sub amostra de $50 \%$ em 1983 e introduzindo a estratificação por faixa etária em 1985 e 1986. Desta forma, as crianças pertencentes ao Inquérito Nutricional eram as dos domicilios sorteados aleatoriamente, pertencentes aos setores e às cidades da amostra.

Em 1983, os levantamentos: antropométrico, clínico e dietético foram realizados nas seis cidades já referidas. Em 1985 e 1986 ocorreu na zona urbana de Cáceres que por suas caracteristicas geográficas representa o ecossistema do Pantanal Matogrossense. Nestes dois últimos foram realizados o estudo antropométrico e do consumo alimentar.

\section{ANTROPOMETRIA}

A situação nutricional das crianças foi determinada através das medidas de peso e estatura combinadas para se obter os seguintes indicadores: a) peso/idade; b) altura/idade; c) peso/altura. Estes indicadores foram classificados segundo os critérios de Gomez para prevalência e intensidade da desnutrição. Para definir as formas da desnutriçào: atual, crônica e pregressa utilizaram-se os critérios de Waterlow.

\section{EXAME CLÍNICO-NUTRICIONAL}

Este exame baseia-se no exame de certas alteraçôes encontradas na morfologia dos tecidos epiteliais externos. A identificação destes sinais em sua forma discreta, apesar da dificuldade do diagnóstico diferencial, permite a identificação de carência nutricional antes das mesmas atingirem formas avançadas.

\section{CONSUMO DE ALIMENTOS}

$\mathrm{Na}$ investigação de 1983 considerou-se o estudo sobre o consumo de alimentos como um levantamento inicial no sentido de elucidar as questões básicas sobre o universo alimentar da população em referência para, em conjunto, com outras análises contribuir para a explicaçào da questão nutrição/desnutrição:

Para a coleta dos dados adotou-se o método recordatorio de 24 horas, aplicado pelos mesmos entrevistadores que realizaram a Entrevista Domiciliar.

Nos levantamentos de 1985 e 1986 , com finalidade de aprofundar a análise sobre as condiçòes nutricionais das crianças e o processo de instalação da desnutrição e investigação do consumo de alimentos limitou-se ao grupo de crianças de 6 a 24 meses de idade, levantando dados sobre a amamentação, o desmame e a introdução de alimentos e, também, sobre a alimentaçào recebida no dia anterior à entrevista. As entrevistas foram realizadas por nutricionistas junto às mães ou responsáveis pelas crianças incluidas na amostra.

\section{DADOS SÓCIO-ECoNôMICOS}

As informações sobre as características sócio-econômicas foram retiradas dos formulários aplicados pela Entrevista Domiciliar.

\section{RESULTADOS}

Procedendo-se a caracterização das famílias estudadas no Inquérito Nutricional em 1985, e no intuito de ampliar a compreensão do processo nutrição e desnutrição trabalhou-se as seguintes variáveis:

- condiçôes;

- composição familiar;

- procedencia do chefe da família;

- ocupaçào e posição na ocupação do chefe da tamilia;

- renda monetária do chefe da família.

Quanto às condições sanitárias de habitacão observou-se que $80 \%$ das famílias estudadas utilizam água encanada, 16\% utilizam fos- 
SANTOS SPINELLI, M.A. dos; SILVA, R.R. da; ALBUQUERQLE, M.C.W; YOKOO, E.M. \& GUIMARÄES, L.V. - Indicadores das condiçðes nutricionais na Região do Polonoroeste. I. Aspectos metodológicos e características socio-econônicas. Rev. Inst. Med. trep. São Paulo, 30(3):169-173, 1988

sa septica e outras $56 \%$ utilizam fossa asséptica ou fossa seca. A coleta pública de lixo atinge $26 \%$ das familias pesquisadas.

A partir de dados sobre a composição familiar, encontrou-se que $77,4 \%$ das famílias eram do tipo nuclear (composta por pai, màe e filhos) e que $22,6 \%$ eram do tipo ampliada (além do núcleo familiar contavam com outros familiares e/ou agregados).

A tabela 1 apresenta a distribuição das familias estudadas de acordo com a procedência do chefe da família. Verifica-se que $74,0 \%$ eram oriundas do próprio município de Cáceres.

\section{TABELA 1}

Local de procedência dos chefes das famílias estudada Cáceres -1985 .

\begin{tabular}{lc}
\hline Local & \multicolumn{1}{c}{$\%$} \\
\hline Outro País & 0,5 \\
Outra Região do País & 13,0 \\
Estado de Mato Grosso & 12,5 \\
Muncípio de Cáceres & 74,0 \\
\hline Total & 100,0 \\
\hline
\end{tabular}

Com relação ao item procedência, os dados demonstram que $76,2 \%$ são de origem urbana.

A tabela 2 apresenta a distribuição dos chefes das famílias observadas de acordo com a categoria social ${ }^{*}$ e a renda dos chefes das famílias estudadas, a partir da sua ocupação e inserção no processo produtivo.

Verifica-se que $51,0 \%$ dos chefes têm renda menor que 2 salários mínimos, percentual que se eleva para $54,0 \%$ se consideram os desempregados.

Há predominio da categoria assalariado urbano $(52 \%)$. Nesta categoria estão inseridos a pequena burguesia gerencial que assume a posição de mando e de controle de um dado processo econômico cujos salários correspondem a maiores valores, os assalariados propriamente ditos e, também, o sub-proletariado com empregos incertos, ocupações mal definidas e menores rendimentos.

(“) Para a classificação das familias por categorias sociais tomou-se como referência o "Esquema Geral da Composiçá das Frações de classe social". proposto por Barroš e baseada na análise de Singer ${ }^{6}$.
TABEIA 2

Categoria Social e Renda dos Chetes das familias Estudadas -. Cáceres - 1985.

\begin{tabular}{|c|c|c|c|c|c|}
\hline \multirow{2}{*}{$\begin{array}{l}\text { Categoria } \\
\text { Social }\end{array}$} & \multicolumn{4}{|c|}{ Renda (Em Salários Mínimos) } & \multirow{2}{*}{$\begin{array}{c}\text { Lotal } \\
\%\end{array}$} \\
\hline & $<2$ & $\begin{array}{l}2-5 \\
\%\end{array}$ & $\begin{array}{l}>5 \\
\%\end{array}$ & $\begin{array}{c}\mathrm{SI} / \mathrm{SR}^{*} \\
\%\end{array}$ & \\
\hline \multicolumn{6}{|l|}{ Assalariados } \\
\hline Urbanos & 28,0 & 18,0 & 6,0 & -. & 52,0 \\
\hline Autônomos & 6,0 & 7,0 & -. & -. & 13,0 \\
\hline Empregadores & - & $\ldots$ & 5.5 & -. & 5,5 \\
\hline $\begin{array}{l}\text { Proprietários } \\
\text { (Agropecuária) }\end{array}$ & - & - & $\cdots$ & 2.0 & 2,0 \\
\hline Assalariados & & & & & \\
\hline Rurais & 10,5 & 2,5 & 0,5 & - & 13,5 \\
\hline Desempregados & - & - & - & 3,0 & 3,0 \\
\hline Outros & 6,5 & 1,0 & 0.5 & 3,0 & 11,0 \\
\hline Total & 51,0 & 28,5 & 13,0 & 8,0 & 100,0 \\
\hline
\end{tabular}

* Sem informação/sem renda.

Para categorizar os empregos considerouse apenas o numero de empregns e o zondimento mensal declarado.

$\mathrm{Na}$ categoria assalariados do camno estão incluidos os administradors de propriedades rurais (que apresentaram rendimentos de até 5 salários-mínimos) e os diaristas e mensalistas.

No grupo "Outros" estão incluidos os pescadores, garimpeiros, desempregados, aposentados e parte dos trabalhadores do campo.

\section{DISCUGSÃO}

Devido a não inclusa neste levantamento dos 2 setores mais pobres e populosos da cidade, onde, certamente, se encontram os grupos estabelecidos há um menor tempo na região, estes dados refletem a realidade dos grupos estabelecidos nos setores centrais e intermediários da cidade que conta com certa condição de infra-estrutura de serviços, como o abastecimento de água.

Rendas maiores que 10 salários-mínimos foram referidas entre os assalariados urbanos (pequena burguesia gerenciai) e entre os empregadores, enquanto que entre os autônomos foram referidas rendas inferiores a 5 salários mínimos o que pode levar à ideia de que estes se confundem com o sub-proletariado urbano.

O grupo que, provavelmente, representa a reserva de mão-de-obra para o capitai aporece na Tabela 2 sob o item "Outros" aos quais 
SANTOS SPINELLI, M.A. dos; SILVA, R.R. da; ALBUQUERQUE, M.C.W.; YOKOO; E.M. \& GUIMARÃES, L.V. -- Indicadores das condições nutricionais na Região do Polonoroeste. I. Aspectos metodologicos e características sócio-econômicas. Rev. Inst. Med. trop. São Paulo, 30(3):169-173, 1988.

pode-se agregar os desempregados o que perfaz $14 \%$ dos chefes das famílias.

Se considerarmos, ainda, que o item assalariados rurais não retrata o tipo de contrato e de vínculo empregatício que regem as suas condições de trabalho, é bem possível que parte desses trabalhadores também sejam reserva de mão-de-obra.

Quanto à renda, estes dados demonstram ainda uma distorção com relação à população brasileira como um todo, onde cerca de $80 \%$ do total aufere renda abaixo de 2 salários mínimos, enquanto entre famílias estudadas, $51 \%$ dos chefes de família recebem até 2 salários mínimos, mais uma vez devemos referir que esta diferença se deve, certamente, a não inclusão de 2 setores na amostra.

\section{SUMMARY}

Nutritional conditions indicators in Polonoroeste Area. I. Methodological aspects and social-economic characteristics

The work presents the methodological principles which lead Nutritional inquires Survey of Polonoroeste/MT organization. Were, also, included the informations about the socialeconomic and sanitary characteristics, of families studied in 1985, in the Cáceres/MT urban zone.

\section{REFERÊNCIAS BIBLIOGRÁFICAS}

1. ALBUQUERQUE, M.C.W.; SILVA, R.R. da; SPINELLI, M.A. dos S.; TEIXEIRA, I.R.; SANTOS, S.M.C. dos; YOKOO, E.M.; MEIRELLES, S.M.P. \& MARCHINI, J.S. - Inquérito Nutricional em JauruMT. Rev. Univ. Fed. Mato Grosso, 4(3): 109-135, 1984.

2. BARROS, M.B. - A utilização do conceito de classe social nos estudos dos perfis epidemiológicos: uma proposta. Rev. Saúde públ. (S. Paulo), 20: 269-273, 1986.

3. MEIRELLES, S.M.P. - Fundamentos e estrut uração de pesquisa em Saúde Pública no Polonoroeste, em Mato Grosso. Rev. Univ. Fed. Mato Grosso, 4(3): 9-18, 1984

4. MONTEIRO, C.A. - Os determinantes da desnutrição infantil no Vale do Ribeira. Cadern. pesq. (Fund. Carlos Chagas) (29): 57-75, 1979.

5. PROGRAMA do Polonoroeste/Segmento Ciência e Tecnologia - Sub-projeto ecologia humana. Primeiro documento síntese: formulação dos problemas básicos de ecologia humana na região do Polonoroeste-MT. Cuiabá, 1983. (mimeografado).

6. SANCHES, O. \& CARVALHEIRO, J.R. - Descrição do desenho da amostra da pesquisa em Saúde Pública no Polonoroeste-M'T. Rev. Univ. Fed. Mato Grosso, 4(3): 87-95, 1984. 